3 D'amico DJ, Skolnik PR, Kosloff BR, Pinkston P, Hirsch MS, Schooley RT. Resolution of cytomegalovirus retinitis with zidovudine cytomegalovirus retinitis with zidovudin

4 Guyer DR, Jabs DA, Brant AM, Beschorner WE Green WR. Regression of cytomegalovirus retinitis with zidovudine. A clinicopathological correlation. Arch Ophthalmol 1989;107:868-74

5 Carter JE, Shuster AR. Zidovudine and cytomegalovirus retinitis. Ann Ophthalmol 1992;24: 186-9.

6 Masur H, Ognibene FP, Yarchoan R, Shelhamer $\mathrm{JH}$, Baird F, Travis W, et al. CD4 counts as predictors of opportunistic pneumonias in human immunodeficiency virus (HIV) infection. Ann intern Med 1989;111: 223-31.

7 Kuppermann BD, Petty JG, Richman DD Mathews WC, Fullerton SC, Rickman LS, et al. Correlation between $\mathrm{CD} 4+$ counts and prevalence of cytomegalovirus retinitis and human immunodeficiency virus-related noninfectious retinal vasculopathy in patients with acquired immunodeficiency syndrome. Am $\mathcal{f}$ Ophthalmol 1993;115:575-82.

8 Lowder CY, Butler CP, Dodds EM, Secic M Recillas-Gispert C. CD8+ T lymphocytes and cytomegalovirus retinitis in patients with the acquired immunodeficiency syndrome. $A m$ acquired immunodeficiency
Ophthalmol 1995;120:283-90.

\section{Bilateral cytomegalovirus retinitis in a patient with systemic lupus erythematosus}

EDIToR,-Cytomegalovirus (CMV) retinitis is common in patients with AIDS. In other immunocompromised hosts this devastating eye condition is much rarer despite the frequent systemic CMV infections in these patients.

We report a patient with systemic lupus erythematosus (SLE) presenting with bilatera CMV retinitis and retinal detachment. $\mathrm{Re}-$ markably, active SLE or manifest systemic CMV infection were absent. Our patien responded well to reduction of the immunosuppressive therapy, ganciclovir therapy, and surgery. We conclude that clinicians should be aware of the possibility of CMV retinitis in rheumatological patients on mild immunosuppression.

\section{CASE REPORT}

A 27-year-old woman presented in March 1993 with decreased vision, floaters, and photopsia of the left eye for a few days. From the age of 5 she has suffered from SLE with chronic immune complex mediated nephritis. In September 1992 a severe exacerbation of SLE complicated by nephritis, pericarditis, and cardiac failure was treated with nine intravenous pulse doses of $1000 \mathrm{mg}$ methylprednisolone and $100 \mathrm{mg}$ azathioprine daily. In 1977, both eyes had suffered from sever SLE retinopathy with widespread vasculopathy resulting in poor vision of the right eye.

On presentation in March 1993 visual acuities were $1 / 60$ in the right eye and $6 / 9$ in the left eye. No signs of inflammation in the anterior segment or vitreous were present in eithe eye. Fundus examination showed right optic nerve pallor, severe bilateral venous frosted branch sheathing (Fig 1), and large areas of white necrotic retina with haemorrhages in the right nasal periphery and left upper retina (Figs 1 and 2). The peripheral retina of the left eye was detached (Fig 2) and showed severa atrophic breaks. Physical examination and laboratory investigations (anti-ds DNA and complement factors $\mathrm{C} 3, \mathrm{C} 4$, and $\mathrm{C} 1 \mathrm{q}$ ) indicated that SLE was in remission. Leucocyte and lymphocyte counts were normal.

Her medication at this time consisted of oral prednisone $20 \mathrm{mg}$ and azathioprine 50

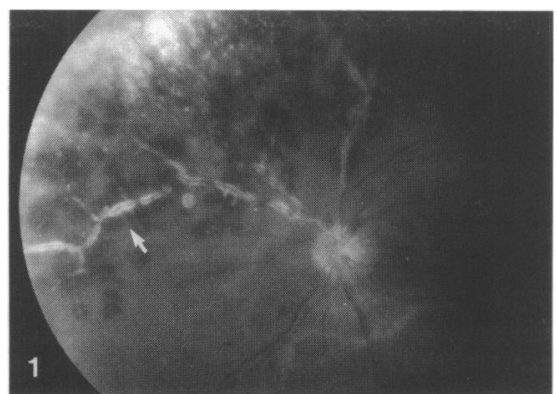

Figure 1 Left eye demonstrates extensive sheathing of retinal venules (frosted branch vasculitis, indicated by arrow) in the posterior pole and classic CMV retinitis in the upper peripheral retina.

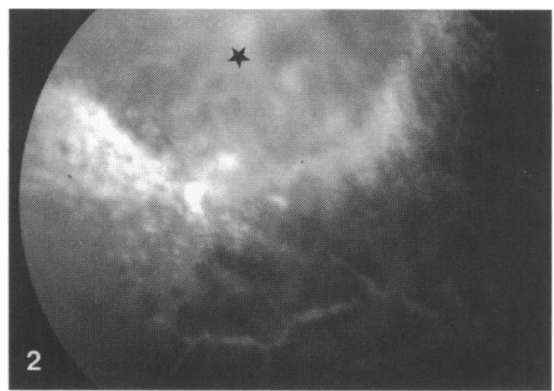

Figure 2 Upper peripheral retina of left eye demonstrates a large area of typical $C M V$ retinitis with retinal detachment (indicated by asterisk).

mg daily. The azathioprine was stopped, the prednisone slowly tapered, and treatment with foscarnet $200 \mathrm{mg} / \mathrm{kg}$ daily was started. The next day the left retinal detachment was treated with vitrectomy, scleral buckling, cryotherapy, and silicone oil tamponade and a prophylactic scleral buckling procedure was performed on the right eye. Analysis of the left vitreous revealed intraocular production of anti-CMV antibodies (Goldmann-Witmer coefficient 4.0) and a polymerase chain reaction (PCR) positive for CMV. PCR reactions for herpes simplex virus, varicella zoster virus, Epstein-Barr virus, and Toxoplasma gondii and tests for local antibody production against these agents were all negative. Because of impaired kidney function the foscarnet was stopped and intravenous ganciclovir was started and maintained for 6 weeks until the retinitis had been inactive for 3 weeks. Visual acuity of the left eye remained $6 / 9$ after removal of the silicone oil. No antiviral maintenance therapy was given and in the next 3 years of regular follow up visits no recurrence of retinitis has occurred nor has her general health changed. She remained on $10 \mathrm{mg}$ prednisone a day.

\section{COMMENT}

The diagnosis of CMV retinitis was established in our patient by its typical aspect and associated frosted branch vasculitis, ${ }^{1}$ and the demonstration of CMV by PCR and intraocular anti-CMV antibodies. Remarkably, at the time of CMV retinitis, the SLE in this patien was inactive and her immunosuppressive medication was mild, with normal leucocyte and lymphocyte counts. Furthermore, she lacked other signs of systemic CMV infection. This clinical picture is different from the severe systemic CMV infections, often with a fatal course, that are common in immunosuppressed SLE and other rheumatic patients and transplant recipients, ${ }^{2-9}$ particularly in those treated with azathioprine or cyclophosphamide. ${ }^{9}$ In fact, such patients only rarely show concurrent CMV retinitis. ${ }^{26-9}$

Our patient's presentation with bilateral severe CMV retinitis, without systemic signs, more resembled CMV infections as seen in patients with AIDS. ${ }^{10}$ AIDS in our patient could not be excluded as she refused an HIV test. However, the lack of recurrent CMV retinitis or other opportunistic infections and her survival in the 3 years of follow up make it unlikely that she has AIDS.

No clear clinical guidelines are established for treating patients on immunosuppressive therapy who develop isolated CMV retinitis. A reduction in immunosuppressive medication may be sufficient in some cases (C Pavesio, personal communication). Others, like our patient, may also need a course of ganciclovir and surgical therapy. In our opinion, maintenance ganciclovir therapy, as is given to AIDS patients, ${ }^{10}$ is not necessary in this patient group. ${ }^{9}$

We conclude that severe $\mathrm{CMV}$ retinitis without systemic symptoms may develop in patients with quiescent SLE on mild immunosuppression and that clinicians should be aware of visual symptoms in these patients.

R O SCHLINGEMANN

Department of Ophthalmology, Academic Medical Center, University of Amsterdam

P WERTHEIM-VAN DILLEN

Department of Medical Microbiology, Academic Medical Center, University of Amsterdam

A KIJLSTRA

The Netherlands Ophthalmic Research Institute

P J M BOS

Department of Ophthalmology, Academic Medical Center, University of Amsterdam

C MEENKEN

Department of Ophthalmology, Academic Medical Center, University of Amsterdam

E J FERON

Rotterdam Eye Hospital, Rotterdam, the Netherlands

Correspondence to: Dr R O Schlingemann, Department of Clinical Ophthalmology, Moorfields Eye Hospital, City Road, London EC1V 2PD.

Accepted for publication 26 August 1996

1 Rabb MF, Jampol LM, Fish RH, Campo RV, Sobol WM, Becker NM. Retinal periphlebitis in patients with acquired immunodeficiency synpatients with acquired immunodeficiency syn-
drome with cytomegalovirus retinitis mimics drome with cytomegalovirus retinitis mimics acute frosted retinal peri
mol 1992;110:1257-60.

2 Bowness P, Davies KA, Norsworthy PJ, Athanassiou P, Taylor-Wiedeman J, Borysiewicz LK, et al. Hereditary $\mathrm{Clq}$ deficiency and systemic lupus erythematosus. $Q f M \quad 1994 ; 87: 455-64$.

3 Bulpitt KJ, Brahn E. Systemic lupus erythematosus and concurrent cytomegalovirus vasculitis: diagnosis by antemortem skin biopsy. $¥$ Rheumatol 1989;16:677-80.

4 Bresser P, Toben FM, van Son WJ, Anema J, Beukhof JR. Current developments in the diagnosis and therapy of cytomegalovirus infections. Ned Tijdschr Geneeskd 1989;133:455-7.

5 Iwasaki T, Satodate R, Masuda T, Kurata T, Hondo R. An immunofluorescent study of generalized infection of human cytomegalovirus in a patient with systemic lupus erythematosus. Acta Pathol fpn 1984;34:869-74.

6 Egbert PR, Pollard RB, Gallagher JG, Merigan TC. Cytomegalovirus retinitis in immunosuppressed hosts. II. Ocular manifestations. Ann pressed hosts. II. Ocular

7 Scott WJ, Giangiacomo J, Hodges KE. Accelerated cytomegalovirus retinitis secondary to immunosuppressive therapy. [Letter] Arch Ophimmunosuppressive therapy.

8 Winkler A, Finan MJ, Pressly T, Roberts R. Cytomegalovirus retinitis in rheumatic disease a case report. Arthritis Rheum 1987;30:106-8. 
9 Rubin RH. Impact of cytomegalovirus infection on organ transplant recipients. Rev Infect Dis 1990;12(Suppl 7):S754-66.

10 Dhillon B. The management of cytomegalovirus retinitis in AIDS. Br f Ophthalmol 1994;78:669.

\section{Optic neuritis following measles/rubella} vaccination in two 13-year-old children

EDrToR,-During November 1994 following the Joint Committee on Vaccination and Immunisations recommendations a widespread school based immunisation programme was set up for measles and rubella. The objectives were to negate the need for a single antigen rubella vaccine to be given to 11-year-old girls and to promote herd immunity to measles.

We describe two 13-year-old children who developed optic neuritis 2-3 weeks after measles/rubella vaccination.

\section{CASE 1}

A 13-year-old boy of Asian origin was seen on the 23 December 1994 with a 2-3 week history of deteriorating vision. Initially this began in the left eye but was bilateral within a few days and was associated with photophobia and pain on eye movements. Three weeks before the symptoms appeared he had received a measles/rubella vaccination at school. There was no family history of Leber's optic neuropathy, multiple sclerosis, or other neurological or ocular disease.

General and neurological examination excluding the ocular findings was normal. Visual acuities were corrected to right eye $1 / 60, \mathrm{~N} 14$, and left eye $6 / 36$, N8. Ishihara plates for colour vision were right eye $1 / 17$ and left eye $4 / 17$. Visual fields demonstrated bilateral central scotomas. Funduscopy revealed mild bilateral optic disc hyperaemia with a single flame-shaped peripapillary haemorrhage on the left. The peripheral retina, macula, vitreous anterior segments, and extraocular movements were normal for each eye.

Investigations revealed normal full blood count, biochemistry profile, clotting studies, erythrocyte sedimentation rate, vitamin $\mathrm{B} 12$, and serum angiotensin converting enzyme. Autoantibodies, the VDRL test, and heavy metal screen were negative. Magnetic resonance imaging (MRI) (axial T2 and coronal T1 cuts, no contrast) showed mild signal change in the posterior part of the optic nerves and chiasm; this was felt to be consistent with inflammation or demyelination. There were no white matter lesions elsewhere in the brain. Fundus fluorescein angiography demonstrated mild tortuosity of the retinal vessels but there was no disc leakage or evidence of subclinical periphlebitis. CSF examination revealed normal constituents; oligoclonal bands were negative. Tests for the pathological mutations of mitochondrial DNA associated with Leber's hereditary optic neuropathy (LHON) at the 11778,3460 , and 14484 sites were all negative.

The patient was treated with vitamin B12 injections and intravenous methylprednisolone but made no recovery. On review in September 1995 visual acuities were $6 / 60$ and N48 in both eyes with bilateral central scotomas and pale discs. Repeat MRI (without contrast) of the brain and orbits was normal.

\section{CASE 2}

A 13-year-old girl received the measles/rubella vaccination in December 1994. Eighteen days later she developed, over a 24-48 hour period, blurring of vision with associated pain on ocular movement in the left eye. There were no other neurological or systemic symptoms and no family history of neurological or ocular disease.

General examination was normal. Visual acuities were corrected to right eye $6 / 6, \mathrm{~N} 4.5$, and left eye $1 / 60$. Ishihara plates for colour vision were right eye $17 / 17$ and left eye $0 / 17$. There was a relative afferent pupillary defect on the left. Visual fields demonstrated normal fields on the right but a large central scotoma on the left. Funduscopy revealed a hyperaemic and swollen left optic disc. The peripheral retina, macula, vitreous anterior segments, and extraocular movements were normal in each eye. Investigations revealed a normal full blood count, biochemistry profile, and erythrocyte sedimentation rate. The serum angiotensin converting enzyme was mildly elevated at 68 (reference range 16-53), serum calcium and chest $x$ ray were normal. The autoantibodies and VDRL tests were negative. MRI (T2 weighted axial and T1 weighted coronals, no contrast) showed mild asymmetry of the optic nerves only. There were no white matter lesions in the brain. CSF examination revealed normal constituents; the oligoclonal bands were positive. Virology 6 weeks after the measles/rubella vaccination showed no detectable measles or rubella IgM but a serum measles IgG titre of 1:160.

The patient was treated with intravenous methylprednisolone and made some recovery. On review in April 1995 visual acuities were right eye $6 / 5$ and left eye $6 / 12$, Ishihara plates right eye $17 / 17$ and left eye $2 / 17$. Funduscopy revealed left optic atrophy.

\section{COMMENT}

Neurological complications following rubella or measles vaccination are rare. Rubella has been associated with carpal tunnel syndrome, paraesthesias, myelitis, and myeloradiculitis. Measles vaccination has been linked to cases of encephalitis, encephalopathy, febrile convulsions, and encephalomyelitis but these are extremely rare. It must be remembered that the risk of encephalitis following measles infection is one per 1000 cases and the incidence of all reported neurological disorders following measles vaccination is only 1.16 per million doses. This compares with the background incidence of encephalitis not related to immunisation of 2 to 3 per million children of similar age. ${ }^{23}$ There is certainly no doubt that the risks of serious neurological disorders are much greater with the natural disease than after the vaccination and widespread immunisation programmes are justified by the available evidence.

The onset of symptoms is usually 1-3 weeks after vaccination, the period when virus replication is maximal and when viraemia is expected to occur. The mechanism is thought to be due to immune complex mediated vascular injury causing alterations in vascular permeability, perivascular inflammation, and consequent blood-brain barrier impairment. This may allow lymphocytes committed to specific viral antibody synthesis outside the brain compartment to enter the brain producing inflammation and demyelination. ${ }^{24}$ It has been suggested that viral infection or vaccination with live or inactivated viruses are often preceding events in optic neuritis; however, population based studies have shown no such association for influenza vaccines. ${ }^{5}$

Optic neuritis has been reported as a complication with the use of several vaccines including rabies, smallpox, swine flu, diphtheria, tetanus, and BCG, although it is extremely rare. There have only been three cases of optic neuritis following immunisation against rubella or measles reported to date. The first was by Kazarian and Gager in $1978,{ }^{6}$ who described a 6-year-old boy developing bilateral simultaneous optic neuritis (BSON) 18 days after measles/mumps/rubella vaccination; the second, by Kline et $a l^{7}$ reported a 31-year-old woman who developed BSON and myelitis 11 days after monovalent rubella vaccination. A third patient, mentioned by Riikonen, ${ }^{8}$ developed unilateral optic neuritis, and later multiple sclerosis, 4 weeks after rubella vaccination.

In the first two reported cases visual recovery was good, in contrast with our patient with BSON who is now registered partially sighted. Optic neuritis in childhood, which is usually bilateral and often associated with a febrile illness, has an excellent prognosis with visual acuities often returning to normal within 4-6 weeks of onset, making our first case unusual. The frequency of later development of multiple sclerosis after optic neuritis in childhood appears to be low and after BSON is extremely rare. ${ }^{910}$

The differential diagnosis in our first patient must include LHON despite the early age of onset, the eyes being affected simultaneously, and the negative family history. Many atypical cases of LHON are now recognised and the phenotype is expanding as a consequence of mitochondrial DNA mutation testing ${ }^{11}$; however, our patient is negative for the described DNA mutations. The presence of positive oligoclonal bands in the second case is indicative of local synthesis of IgG typical of demyelination but is also seen as a response to the presence of viral antigens.

The association between immunisation and optic neuritis has now been described on several occasions and there is certainly biological plausibility in the suggested pathogenesis. However, the number of reported cases are too small for a definite causal relation to be defined at present. ${ }^{12}$

V L STEVENSON NMR Research Group, Institute of Neurology, Queen Square, London

J F ACHESON J BALL

GT PLANT

National Hospital for Neurology and Neurosurgery, Queen Square, London

Correspondence to: $\mathrm{Dr}$ VL Stevenson, NMR Research Group, Institute of Neurology, Queen Square, London WC1N 3BG.

Accepted for publication 13 September 1996

1 Miller E. The new measles campaign. $B M \mathcal{F}$ 1994;309:1102-3.

2 Landrigan PJ, White JJ. Neurological disorders following live measles-virus vaccination. $\mathfrak{f} A M A$ 1973;223:1459-62.

3 Fenichel GM. Neurological complications of immunization. Ann Neurol 1982;12:119-8.

4 Reik L. Disseminated vasculomyelinopathy. An immune complex disease. Ann Neurol 1981;7: 291-6.

5 Salvetti M, Pisani A, Bastianello S, Millefiorini E Buttinelli C, Pozzilli C. Clinical and MR assessment of disease activity in patients with multiple sclerosis after influenza vaccination. $\mathcal{f}$ Neurol 1995;242:143-6.

6 Kazarian EL, Gager WE. Optic neuritis complicating measles, mumps and rubella vaccination. Am f Opthalmol 1978;86:544-7. 